\title{
Associations between alcohol use disorders and adherence to antiretroviral treatment and quality of life amongst people living with HIV/AIDS
}

\author{
Bach Xuan Tran ${ }^{1 *}$, Long Thanh Nguyen ${ }^{2}$, Cuong Duy Do ${ }^{3}$, Quyen Le Nguyen ${ }^{4}$ and Rachel Marie Maher ${ }^{1}$
}

\begin{abstract}
Background: We examined the association of alcohol use disorders (AUD) with adherence to and health-related quality of life (HRQOL) outcomes of antiretroviral treatment (ART) for HIV/AIDS patients.

Methods: A cross-sectional multi-site survey was conducted in 468 drug users and 648 non-drug users (age: $35.4 \pm$ 7.0 years; $63.8 \%$ male) in 3 epicentres of Vietnam. AUD, ART adherence, and HRQOL were measured using the Alcohol Use Disorders Identification Test - Consumption (AUDIT-C), the self-reported Visual Analogue Scale (VAS), and the World Health Organization Quality of Life instrument (WHOQOL-HIV BREF).

Results: $35.0 \%$ of drug users were hazardous drinkers, compared to $25.9 \%$ of non-drug users. $22.3 \%$ of drug users engaged in binge drinking, and 25.9\% reported suboptimal ART adherence. Adjusting for propensity scores of AUD, patients who had either at-risk or binge drinking behaviour were about twice as likely to be treatment non-adherent as those who did not have AUD. Hazardous drinkers reported small to medium decrements in the Performance, Physical, Social, Spirituality, and Environment quality of life domains. Binge drinkers had a slightly higher score in Social dimension.

Conclusion: AUD is prevalent and negatively affecting adherence to and HRQOL outcomes of ART services in injection-driven HIV epidemics. Screening and intervention are recommended for AUD, especially during the stable periods of ART. Other social and psychological interventions might also enhance patients' responses to and outcomes of ART in Vietnam.
\end{abstract}

Keywords: Alcohol use disorders, HIV/AIDS, Antiretroviral treatment, WHOQOL-HIV, Adherence, Vietnam

\section{Background}

In many Asian populations, hazardous alcohol use is found to be associated with the spread of HIV infection and substantial unfavourable outcomes of HIV/AIDS treatment [1-4]. At-risk drinkers are more likely to engage in unprotected sex, which contributes to the transmission of HIV and other sexually transmitted infections [1]. Among HIV/AIDS patients, hazardous drinkers adhered less to antiretroviral treatment (ART) than other patient groups, resulting in poorer immunological and virological treatment outcomes [5]. Alcohol use is also found to be associated with lipodystrophy and exacerbate antiretroviral

\footnotetext{
* Correspondence: bach@hmu.edu.vn

'Institute for Preventive Medicine and Public Health, Hanoi Medical University, Hanoi, Vietnam

Full list of author information is available at the end of the article
}

therapy - induced neuropathic pain in patients with HIV/ AIDS [6,7]. Moreover, it has a direct association with depression and HIV disease progression [8]. Interventions for individuals with substance abuse - including alcohol are therefore necessary measures to control the spread and reduce the impact of HIV/AIDS.

The HIV epidemics in Asia are largely driven by drug injection, and more than half of all people living with HIV/AIDS in these countries are injection drug users [4]. Treatment of opiate drug use during ART has been implemented in some settings, such as the integration of methadone maintenance with ART services $[9,10]$. However, while a high prevalence of alcohol use disorders (AUD) has also been observed among drug users, its negative impact on the outcomes of ART is not fully recognized [11-16]. This lack of knowledge may have a

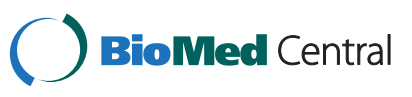


couple of possible explanations. First, alcohol is a legal commodity which is culturally accepted in many Asian cultures [11]. In addition, few studies in Asia have quantified the impact of AUD on HIV/AIDS treatment outcomes, and empirical evidence of large injection-driven HIV epidemics is still limited.

Vietnam has a concentrated HIV epidemic, which emerged initially in drug using populations. It is estimated that 320,000 people have contracted HIV, $70 \%$ of which are drug users [17]. Antiretroviral treatment services have been rapidly scaled up in the country since 2006, and covered $60 \%$ of patients with HIV who were in need of treatment by 2012 [18]. Previous works have shown various factors that influenced adherence to and outcomes of antiretroviral treatment in the Vietnamese settings [18-23]. This included, for instance, avoidance of HIV testing, deferred antiretroviral treatment, heroin use, lack of social and familial support, stigma and discrimination. Although one third of HIV/AIDS patients are hazardous drinkers, the magnitude of AUD's impacts on HIV treatment outcomes have not been determined, and not any intervention of alcohol use among patients with HIV/AIDS has been implemented [11]. In this study we sought to examine the association of AUD on antiretroviral treatment adherence and health-related quality of life (HRQOL) of HIV/AIDS patients receiving treatment from multiple ART clinics in three epicentres of Vietnam. The study provides a baseline for evaluating effectiveness of potential intervention strategies to reduce alcohol consumption among HIV/AIDS patients.

\section{Methods}

\section{Study design and participant recruitment}

This study was a part of the 2012 HIV Services Users Survey, which was conducted in seven clinics in three epicentres of Vietnam: Ha Noi, Hai Phong, and Ho Chi Minh City. The survey included inpatients and outpatients who were attending ART clinics in three district health centres, three provincial hospitals, and one central hospital. A detailed description of survey design and sampling has been presented elsewhere $[11,24,25]$. In short, we purposively selected facilities based on the following criteria: 1) the sample included central-, provincial- and district-level hospitals or health centres 2) they have been providing ART services, and 3) a sufficient number of HIV/AIDS patients attend each clinic. All HIV-positive inpatients and outpatients who were registering for care or taking ART at selected hospitals were eligible for the study. Since HIVrelated information is confidential, it was not feasible to develop a sample frame. Therefore, we selected patients conveniently, including those who were present at the clinics during the study period, and who gave informed consent to participate in the study, until reaching at least 100 patients per site and 200 patients per clinic at the national level. A total of 1016 patients were selected, including 468 drug users and 548 non-drug users.

\section{Measures and instrument}

Patients were interviewed using a structured questionnaire about their socioeconomic, clinical and behavioral characteristics. Alcohol use consumption was assessed using the Alcohol Use Disorders Identification Test - Consumption (AUDIT-C). It is a brief version of the 10-question AUDIT instrument, which consists of 3 questions: 1) How often do you have a dink containing alcohol?; 2) How many standard drinks containing alcohol do you have on a typical day?; and 3) How often do you have six or more drinks on one occasion? [26,27]. The AUDIT-C score ranged from $0-12$, where 4 or more in men and 3 or more in women are considered active AUD or at-risk drinking. The third question, AUDIT-3, relates to binge drinking and is defined as positive if it receives any positive response [27]. Antiretroviral treatment adherence was selfreported over the past 30 days using a visual analogue scale (VAS) [28]. The VAS score ranged at [0; 100] where the threshold for optimal adherence was defined at 95\% and above.

Patients were asked to complete a questionnaire about their HRQOL using the World Health Organization Quality of Life - HIV Brief Instrument (WHOQOL-HIV BREF). Those patients who were severely ill and who experienced any difficulty in completing the form were interviewed by study administrators. The WHOQOLHIV BREF is a multidimensional profile which includes 31 items covering 6 domains and 2 other general items (Overall HRQOL and General Health) [29,30]. The respondents answered each question using a 5-item Likert scale. Average domain scores were multiplied by four to convert domain scores to the range of $[4,20]$, making it comparable with scores derived from the WHOQOL-100. Development of the Vietnamese version and psychometric properties of WHOQOL-HIV BREF have been presented elsewhere [31,32]. In factor analysis, the items were reclassified into 6 modified domains, including: Performance (10 items), Physical (4 items), Morbidity (5 items), Social (4 items), Spirituality (3 items), and Environment (3 items).

\section{Statistical analysis}

Impact of AUD on ART adherence and HRQOL outcomes were examined in multivariate regression models. Since the number of participants and their observed characteristics might be disproportionate between those patients with and without AUD, estimability of the models had the potential to be biased. To compensate for this, we used propensity score to reduce the preexisting differences to a single dimension [33]. A propensity score is defined as the conditional probability of belonging to the AUD group given a vector of observed 
covariates which summarizes information across potential confounders [34]. Propensity scores of AUD (at-risk drinking and binge drinking) were estimated using logistic regression with predictors including socioeconomic status and HIV-related characteristics of respondents. Co-linearity was examined using the variance inflation factors. A stepwise forward model selection was applied, where variables were included based on the loglikelihood ratio test. We adopted a p-value $<0.1$, and excluded variables at $p$-values $>0.2$. The equations are expressed as follows:

$$
\operatorname{LOGIT}[\mathrm{P}(\mathrm{AUD} \mid \mathrm{SES}, \mathrm{HIV})]=\alpha+\sum_{i} \beta_{1 i} S E S_{i}+\sum_{i} \beta_{2 i} H I V_{i}
$$

Where: SES and HIV represent socio-demographic and HIV-related characteristics of respondents.

$S E S_{i}$ included: sex, age (continuous), educational attainment (high school and above, others), marital status (single, live with spouse or partner, widow(er)/divorced/ separated), employment (unemployed, stable jobs, unstable jobs), religion (Buddhism and others), income per capita (five quintiles).

$H I V_{i}$ included length of time living with HIV, HIV stage, length of ART.

Propensity score is calculated as follows:

$$
\text { PROPENSITY }=\operatorname{Predict}[P(\text { AUD } \mid \text { SES }, \text { HIV })]
$$

Propensity score - adjusted linear and logistic regression analysis were used to determine the associations of at-risk and binge drinking with ART non-adherence and HRQOL.

Since WHOQOL-HIV BREF domain scores raged at $[4,20]$, they actually were left and right censored. Censoring from above and below the WHOQOL-HIV BREF domain scores did not allow us to measure exactly the values which were higher or lower than the range thresholds. Therefore, in multivariate linear regression, we employed censored regression models or Tobit models to estimate linear relationships between AUD and HRQOL [35]. Differences in HRQOL scores between patients with and without AUD were then quantified into Cohen's effect size, which is defined as the magnitude of differences divided by standard deviations of the sample measurements. Since drug use is a potential confounder of the association between AUD and ART adherence and outcomes, we stratified this analysis by history of drug use.

\section{Results}

\section{Characteristics of participants}

The sample population studied was $63.8 \%$ men and $36.2 \%$ women, who had a mean age was $35.4(\mathrm{SD}=7.0)$. $45 \%$ had high school education and above, 64\% lived with their spouses or partners, and $20.4 \%$ had stable jobs. A large proportion of patients in the sample had a history of drug use (46.1\%), and $87 \%$ of them actively used drugs at the time of the study. The mean duration of HIV infection was 5.7 years ( $\mathrm{SD}=3.7$ years) and $88.8 \%$ of patients had been taking ART for an average period of 3.0 years $(\mathrm{SD}=2.1$ years). The distribution of patients by ART duration period was as follows: $1^{\text {st }}$ year (19.3\%), $2^{\text {nd }}$ year $(14.2 \%), 2-4$ years $(26.6 \%)$, and $4-7$ years (28.7\%). 31\% of patients had CD4 count less than 200 cells/ $\mu \mathrm{l}$, and $62.2 \%$ had less than 350 cells/ $\mu \mathrm{l}$.

\section{Alcohol consumption, ART adherence and HRQOL profile}

Of the 1016 respondents, 30.1\% were at-risk drinkers (35.0\% among drug users, and 25.9\% among non-drug users), $22.3 \%$ exhibited binge drinking with six or more drinks on one occasion, $25.9 \%$ patients reported nonadherence to ART. As indicated in Table 1, the percentage of at-risk drinking was higher in patients who were not yet on ART (40.4\%) or who were on their $1^{\text {st }}$ year of ART (35.7\%) than in other patients; meanwhile, there was no significant difference in the percentage of binge drinking across ART periods. The percentage of nonadherence to ART was higher in patients with AUD compared to those without AUD in the periods of 12 years and 4-7 years ART. The average HRQOL domain scores for all 1016 respondents was $12.6(\mathrm{SD}=2.3)$ in Performance, $13.2(\mathrm{SD}=3.1)$ in Physical, $12.7(\mathrm{SD}=3.5)$ in Morbidity, $11.2(\mathrm{SD}=3.3)$ in Social, $12.6(\mathrm{SD}=2.9)$ in Spirituality, and $13.8(\mathrm{SD}=2.8)$ in Environment. In all ART periods, HRQOL domain scores were significantly higher in HIV/AIDS patients without AUD than those with AUD, except Morbidity. Compared to other patients groups, patients who were in the 1st year of ART reported lower HRQOL, especially in the Physical domain (Figure 1).

\section{Associations of AUD with antiretroviral treatment adherence and HRQOL}

Table 2 presents the association of AUD with ART adherence and HRQOL in multivariate analysis. Adjusting for propensity scores of AUD, there were small to medium decrements in five HRQOL domains scores (all except Morbidity) in patients who were hazardous drinkers, ranging from 0.3 (Social) to 0.5 (Environment). Compared to non-DU hazardous drinkers, at-risk drinkers who were also drug users reported a larger decrement in Environment, but a smaller decrement in Spirituality.

Binge drinking predicted HRQOL differently than atrisk drinking. HIV/AIDS patients who had binge drinking behaviour reported better HRQOL in five dimensions: Physical, Morbidity, Social, Spirituality, and Environment. However, the difference was small and statistically significant in only the Social domain. 
Table 1 Alcohol use, adherence and health-related quality of life during ART

\begin{tabular}{|c|c|c|c|c|c|c|c|c|c|c|c|c|}
\hline \multirow[b]{3}{*}{ 1. AUD } & \multirow{2}{*}{\multicolumn{2}{|c|}{ All }} & \multicolumn{10}{|c|}{ Duration of ART } \\
\hline & & & \multicolumn{2}{|c|}{ Not-yet } & \multicolumn{2}{|c|}{$<=1$ year } & \multicolumn{2}{|c|}{$1 ;<=2$ year } & \multicolumn{2}{|c|}{$2 ;<=4$ year } & \multicolumn{2}{|c|}{$4 ;<=7$ year } \\
\hline & Mean & SD & Mean & SD & Mean & SD & Mean & SD & Mean & SD & Mean & SD \\
\hline \multirow[t]{2}{*}{ AUDIT-C Score } & 2.0 & 2.3 & 2.4 & 2.5 & 2.1 & 2.4 & 2.1 & 2.3 & 1.8 & 2.1 & 2.0 & 2.5 \\
\hline & $\mathrm{N}$ & $\%$ & $\mathbf{N}$ & $\%$ & $\mathbf{N}$ & $\%$ & $\mathbf{N}$ & $\%$ & $\mathbf{N}$ & $\%$ & $\mathbf{N}$ & $\%$ \\
\hline At-risk drinking & 306 & 30.1 & 46 & 40.4 & 70 & 35.7 & 43 & 29.9 & 67 & 24.8 & 80 & 27.4 \\
\hline Binge drinking & 227 & 22.3 & 28 & 24.6 & 40 & 20.4 & 33 & 22.9 & 65 & 24.1 & 61 & 20.9 \\
\hline 2. HRQOL Domain scores & AUD & & Mean & SD & Mean & SD & Mean & SD & Mean & SD & Mean & SD \\
\hline \multirow[t]{2}{*}{ Performance } & No & & $12.8^{* * *}$ & 2.2 & $12.3^{* *}$ & 2.5 & $12.8^{* *}$ & 2.1 & $13.2^{* * *}$ & 2.3 & $13.0^{* * *}$ & 2.2 \\
\hline & Yes & & 11.6 & 1.9 & 11.5 & 2.4 & 12.0 & 2.1 & 12.2 & 2.3 & 12.2 & 2.1 \\
\hline \multirow[t]{2}{*}{ Physical } & No & & $13.8^{* *}$ & 2.8 & $12.7^{* *}$ & 3.3 & $13.9^{* *}$ & 2.8 & $13.8^{* *}$ & 2.7 & $13.7^{* * *}$ & 3.0 \\
\hline & Yes & & 12.5 & 3.4 & 11.8 & 3.2 & 12.8 & 3.3 & 12.9 & 3.7 & 12.5 & 2.9 \\
\hline \multirow[t]{2}{*}{ Morbidity } & No & & 12.5 & 3.9 & 12.3 & 3.3 & 12.6 & 3.1 & 12.9 & 3.7 & $13.2^{*}$ & 3.6 \\
\hline & Yes & & 12.5 & 3.7 & 12.2 & 3.4 & 12.9 & 3.3 & 13.1 & 3.5 & 12.6 & 3.5 \\
\hline \multirow[t]{2}{*}{ Social } & No & & $12.0^{* * *}$ & 2.9 & $11.0^{*}$ & 3.4 & $11.6^{* *}$ & 3.4 & $11.6^{* * *}$ & 3.4 & $11.4^{*}$ & 3.3 \\
\hline & Yes & & 10.3 & 3.2 & 10.3 & 3.1 & 10.3 & 3.4 & 10.4 & 3.2 & 10.8 & 3.2 \\
\hline \multirow[t]{2}{*}{ Spirituality } & No & & $12.9^{* * *}$ & 2.9 & $12.5^{* * *}$ & 2.9 & $13.1^{* * *}$ & 2.8 & $13.1^{* *}$ & 2.7 & $13.2^{* * *}$ & 2.8 \\
\hline & Yes & & 11.3 & 2.9 & 11.2 & 2.7 & 11.7 & 2.9 & 12.1 & 3.5 & 11.9 & 2.6 \\
\hline \multirow[t]{2}{*}{ Environment } & No & & $14.2^{* * *}$ & 2.5 & $13.6^{* * *}$ & 2.8 & $14.7^{* * *}$ & 2.5 & $14.4^{* * *}$ & 2.7 & $14.2^{* * *}$ & 2.6 \\
\hline & Yes & & 12.6 & 3.3 & 12.4 & 2.8 & 12.7 & 2.9 & 13.0 & 3.4 & 13.2 & 2.9 \\
\hline 3. ART Adherence & AUD & & & & Mean & SD & Mean & SD & Mean & SD & Mean & SD \\
\hline \multirow[t]{2}{*}{ Mean VAS score } & No & & & & 94.9 & 8.5 & $95.3^{* * *}$ & 7.7 & 94.9 & 7.9 & $96.6^{* * *}$ & 4.8 \\
\hline & Yes & & & & 94.1 & 8.5 & 90.3 & 11.6 & 93.8 & 8.0 & 92.1 & 10.3 \\
\hline \multirow[t]{3}{*}{$\%$ Non-adherence } & & & & & $\mathbf{N}$ & $\%$ & $\mathbf{N}$ & $\%$ & $\mathrm{~N}$ & $\%$ & $\mathbf{N}$ & $\%$ \\
\hline & No & & & & 23 & 21.3 & 18 & $19.2^{* * *}$ & 48 & 24.0 & 34 & $16.4^{* * *}$ \\
\hline & Yes & & & & 17 & 28.3 & 19 & 44.2 & 19 & 28.4 & 26 & 32.5 \\
\hline
\end{tabular}

${ }^{* * *} \mathrm{p}<0.01,{ }^{* *} \mathrm{p}<0.05,{ }^{*} \mathrm{p}<0.1$ (Student-t test were used for comparing 2 means. Chi square tests were used for comparing 2 proportions).

During ART, patients who had AUD (both at-risk and binge drinking) were about twice as likely to be treatment non-adherent as those who did not have AUD. The odds ratio of non-adherence was higher in nondrug users than drug users.

\section{Discussions}

This study found that AUD occurs with a high prevalence in large injection-driven HIV/AIDS epidemics in Vietnam, and supports the existing body of evidence of AUD's negative effect on adherence to and outcomes of ART in such epidemics [36]. Moreover, this study contributes to the understanding of AUD's influences on HRQOL outcomes of ART among HIV/AIDS patients. The magnitude of difference in HRQOL between patients with and without AUD in this study was comparable to a similar assessment in drug users [37]. Here, non-drug users were found to be even more likely than drug users to be non-adherent while engaging in either at-risk or binge drinking. Both hazardous and binge drinking problems strongly predicted non-adherence to
ART; however their associations with dimensions of HRQOL were inconsistent. In different ART periods, hazardous drinking seemed to decrease, while binge drinking remained constant across all periods. Hazardous drinking was associated with small-to-medium decrements in almost all HRQOL dimensions, except Morbidity. Meanwhile, binge drinking was only associated with a small increase in the Social dimension.

This finding that binge drinking remained constant throughout the stages of ART and is associated with improved HRQOL in the Social dimension could be explained by the fact that alcohol use is legally accepted and culturally encouraged in Vietnamese society. Even though patients may perceive improved physical and mental health status during stable periods of ART, they may still have an AUD due to their continued binge drinking behaviour. In the modified WHOQOL-HIV BREF, the Social domain comprises 4 items, namely, social inclusion, financial resources, opportunities for acquiring new information and skills, and opportunities for recreation and leisure activities. Besides the slight 


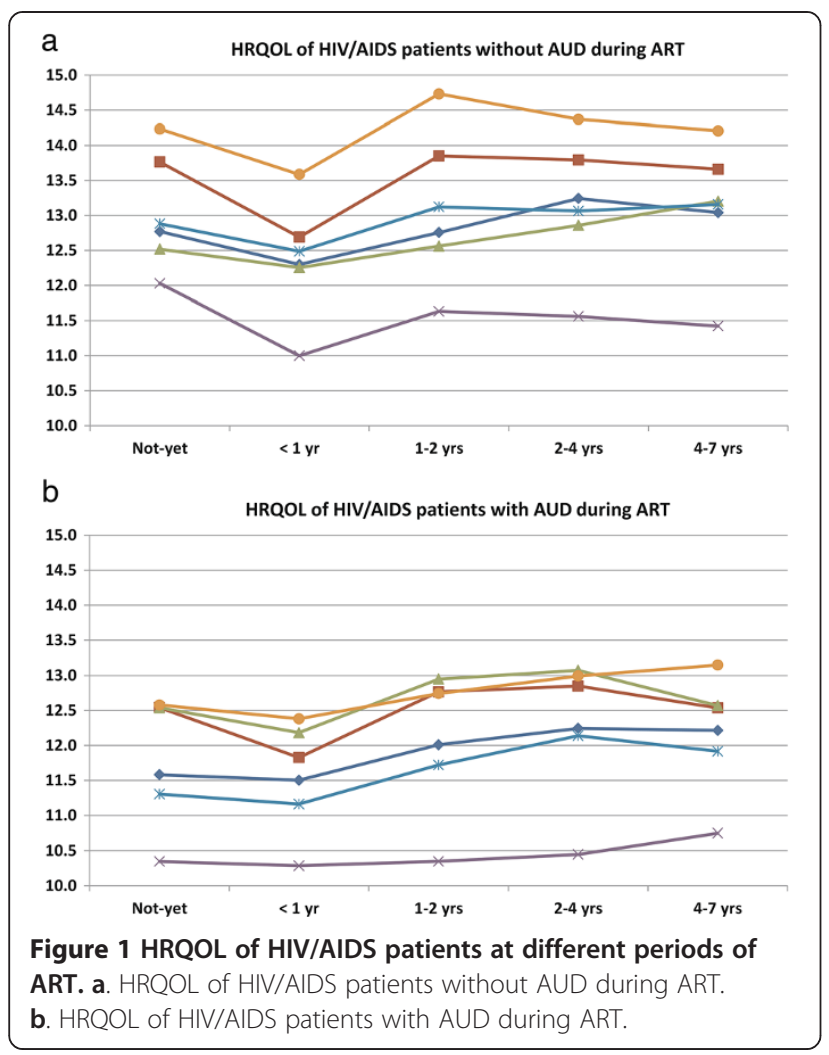

increase among binge drinkers, social functioning was generally the poorest among the six HRQOL dimensions, particularly in at-risk drinkers. This may be explained by the fact that in the concentrated epidemic of Vietnam, the majority of patients have complex social backgrounds - including illicit drug use and sex work that are accompanied by stigma and discrimination [38]. In addition, many HIV/AIDS patients do not have stable jobs, which prevent them from pursuing opportunities to live positively in their fight against HIV/AIDS.

This study's findings suggest important implications for the HIV/AIDS intervention strategy in Vietnam. First, the strong association of AUD with poor adherence to and outcomes of antiretroviral treatment for HIV/AIDS patients highlights the necessity of screenings and interventions for AUD during ART. Given that the prevalence of AUD was high in drug users and non-drug users, such screening and intervention measures should be applied to all patient groups. Second, since adherence is central to achieving viral suppression and preventing drug resistance, intervention for ART adherence should be maintained throughout the stages of ART, and especially during the stable periods, beginning in the second year of treatment. While scaling up ART has substantially relieved the burden of HIV/AIDS in the country, we have found that benefits of ART might be limited if

Table 2 Propensity score-adjusted differences in HRQOL and OR of non-adherence with regard to AUD in HIV/AIDS patients

\begin{tabular}{|c|c|c|c|c|c|c|c|c|c|}
\hline & \multicolumn{3}{|c|}{ All } & \multicolumn{3}{|c|}{ Drug users $(n=468)$} & \multicolumn{3}{|c|}{ Non- drug users $(n=548)$} \\
\hline & Coef. & $95 \% \mathrm{Cl}$ & Effect size & Coef. & $95 \% \mathrm{Cl}$ & Effect size & Coef. & $95 \% \mathrm{Cl}$ & Effect size \\
\hline \multicolumn{10}{|l|}{ 1. HRQOL outcomes } \\
\hline \multicolumn{10}{|l|}{ At-risk drinking vs. None } \\
\hline Performance & $-0.92^{* * *}$ & $(-1.24 ;-0.61)$ & -0.40 & $-0.80^{* * *}$ & $(-1.24 ;-0.36)$ & -0.35 & $-1.07^{* * *}$ & $(-1.52 ;-0.61)$ & -0.46 \\
\hline Physical & $-0.97^{* * *}$ & $(-1.40 ;-0.54)$ & -0.31 & $-0.98^{* * *}$ & $(-1.55 ;-0.40)$ & -0.33 & $-0.97^{* * *}$ & $(-1.60 ;-0.33)$ & -0.31 \\
\hline Morbidity & -0.08 & $(-0.58 ; 0.41)$ & -0.02 & 0.09 & $(-0.60 ; 0.79)$ & -0.03 & -0.26 & $(-0.97 ; 0.44)$ & -0.07 \\
\hline Social & $-0.99 * * *$ & $(-1.45 ;-0.52)$ & -0.30 & $-1.04^{* * *}$ & $(-1.68 ;-0.39)$ & -0.31 & $-0.97^{* * *}$ & $(-1.64 ;-0.29)$ & -0.29 \\
\hline Spirituality & $-1.34^{* * *}$ & $(-1.74 ;-0.94)$ & -0.46 & $-1.04 * *$ & $(-1.56 ;-0.52)$ & -0.38 & $-1.68^{* * *}$ & $(-2.29 ;-1.07)$ & -0.55 \\
\hline Environment & $-1.41^{* * *}$ & $(-1.81 ;-1.02)$ & -0.50 & $-1.70^{* * *}$ & $(-2.24 ;-1.16)$ & -0.60 & $-1.14^{* * *}$ & $(-1.71 ;-0.56)$ & -0.40 \\
\hline \multicolumn{10}{|l|}{ Binge drinking vs. None } \\
\hline Performance & -0.04 & $(-0.40 ; 0.33)$ & -0.02 & 0.16 & $(-0.32 ; 0.65)$ & 0.07 & -0.25 & $(-0.82 ; 0.31)$ & -0.11 \\
\hline Physical & 0.26 & $(-0.23 ; 0.76)$ & 0.08 & 0.25 & $(-0.39 ; 0.89)$ & 0.08 & 0.45 & $(-0.34 ; 1.25)$ & 0.14 \\
\hline Morbidity & 0.32 & $(-0.26 ; 0.89)$ & 0.09 & 0.39 & $(-0.37 ; 1.14)$ & 0.11 & 0.16 & $(-0.73 ; 1.06)$ & 0.05 \\
\hline Social & $0.66^{* *}$ & $(0.12 ; 1.20)$ & 0.20 & 0.48 & $(-0.23 ; 1.19)$ & 0.15 & $0.88^{* *}$ & $(0.04 ; 1.72)$ & 0.26 \\
\hline Spirituality & 0.11 & $(-0.36 ; 0.58)$ & 0.04 & 0.16 & $(-0.40 ; 0.73)$ & 0.06 & -0.02 & $(-0.80 ; 0.76)$ & -0.01 \\
\hline \multirow[t]{2}{*}{ Environment } & 0.23 & $(-0.24 ; 0.69)$ & 0.08 & 0.11 & $(-0.51 ; 0.73)$ & 0.04 & 0.46 & $(-0.27 ; 1.18)$ & 0.16 \\
\hline & OR & $95 \% \mathrm{Cl}$ & & OR & $95 \% \mathrm{Cl}$ & & OR & $95 \% \mathrm{Cl}$ & \\
\hline \multicolumn{10}{|l|}{ 2. ART non-adherence } \\
\hline At-risk drinking vs. None & $2.06^{* * *}$ & $(1.48 ; 2.85)$ & & $1.86^{* * *}$ & $(1.18 ; 2.95)$ & & $2.28^{* * *}$ & $(1.43 ; 3.62)$ & \\
\hline Binge drinking vs. None & $1.97^{* * *}$ & $(1.37 ; 2.82)$ & & $1.57^{*}$ & $(0.96 ; 2.56)$ & & $2.69^{* * *}$ & $(1.55 ; 4.67)$ & \\
\hline
\end{tabular}

${ }^{* * *} p<0.01,{ }^{* *} p<0.05,{ }^{*} p<0.1$. 
other interventions addressing the social and structural barriers associated with HIV/AIDS are not in place. Interventions that improve such aspects of HIV/AIDS patients' lives as spirituality and social functioning might actually hold much potential to support ART adherence and outcomes. Finally, the findings inspire future studies to examine the underlying mechanisms of AUD and ART adherence and outcomes given the sociocultural and epidemiological characteristics of Vietnam.

The strengths of this study included a large sample size across different levels of the health system in 3 epicentres of Vietnam. In addition, we employed validated instruments which ensured improved psychometric properties and comparability of measurements. However, the study has some limitations that should be acknowledged. First, the cross-sectional design may not have allowed for the evaluation of the temporal relationships between AUD, patient adherence to ART, and HRQOL, and was limited in its ability to describe the changes during ART. In addition, the AUDIT-C questions referred to the patient's lifetime drinking experience, thus, might not completely reflect the current behaviour. Self-reported alcohol use and ART adherence was also subject to biases due to patients' recall or influences of health workers. However, comparing to other AUD measures, the AUDIT-C showed very good measurement properties in many studies, including some in Vietnamese populations [26,39-41]. In addition, the VAS for measuring ART adherence had been previously validated in the Vietnamese context, and showed convergent validity with the Adult AIDS Clinical Trials Group instrument [42].

\section{Conclusions}

This study assessed the impact of AUD on ART adherence and HRQOL in HIV/AIDS patients in large injectiondriven HIV epidemics in Vietnam. The magnitude of decrements in HRQOL outcomes suggests that screening and intervening for AUD is needed during ART, particularly during stable periods. Such social and psychological interventions may be extremely important to enhance patients' responses to and outcomes of ART in Vietnam.

\section{Competing interests}

The authors declare that they have no competing interests.

\section{Authors' contributions}

BXT and LTN designed the study and implemeted the survey. BXT analyzed the data. BXT, LTN, CDD, QLN, RMM wrote the manuscript. All authors read and approved the final manuscript.

\footnotetext{
Author details

'Institute for Preventive Medicine and Public Health, Hanoi Medical University, Hanoi, Vietnam. ${ }^{2}$ Authority of HIV/AIDS Control, Ministry of Health, Hanoi, Vietnam. ${ }^{3}$ Department of Infectious Diseases, Bach Mai Hospital, Hanoi, Vietnam. ${ }^{4}$ School of Medicine and Pharmacy, Vietnam National University, Hanoi, Vietnam.
}

Received: 7 March 2013 Accepted: 5 January 2014

Published: 10 January 2014

\section{References}

1. Schneider M, Chersich M, Neuman M, Parry C: Alcohol consumption and HIV/AIDS: the neglected interface. Addiction 2012, 107(8):1369-1371.

2. Mimiaga MJ, Thomas B, Mayer KH, Reisner SL, Menon S, Swaminathan S, Periyasamy M, Johnson CV, Safren SA: Alcohol use and HIV sexual risk among MSM in Chennai, India. Int J STD AIDS 2011, 22(3):121-125.

3. Schensul JJ, Singh SK, Gupta K, Bryant K, Verma R: Alcohol and HIV in India: a review of current research and intervention. AIDS Behav 2010, 14(Suppl 1):S1-7.

4. The Commission on AIDS in Asia: Redefining AIDS in Asia: crafting an effective response. Oxford University Press; 2008:258. Available at http://data.unaids. org/pub/report/2008/20080326_report_commission_aids_en.pdf. Accessed November 1, 2012

5. Neuman MG, Monteiro M, Rehm J: Drug interactions between psychoactive substances and antiretroviral therapy in individuals infected with human immunodeficiency and hepatitis viruses. Subst Use Misuse 2006, 41(10-12):1395-1463.

6. Ferrari LF, Levine JD: Alcohol consumption enhances antiretroviral painful peripheral neuropathy by mitochondrial mechanisms. Eur J Neurosci 2010, 32(5):811-818.

7. Cheng DM, Libman H, Bridden C, Saitz R, Samet JH: Alcohol consumption and lipodystrophy in HIV-infected adults with alcohol problems. Alcohol 2009, 43(1):65-71.

8. Ghebremichael M, Paintsil E, Ickovics JR, Vlahov D, Schuman P, Boland R, Schoenbaum E, Moore J, Zhang H: Longitudinal association of alcohol use with HIV disease progression and psychological health of women with HIV. AIDS Care 2009, 21(7):834-841.

9. Tran BX, Ohinmaa A, Duong AT, Nguyen LT, Vu PX, Mills S, Houston S, Jacobs $P$ : Cost-effectiveness of integrating methadone maintenance and antiretroviral treatment for HIV-positive drug users in Vietnam's injection-driven HIV epidemics. Drug Alcohol Depend 2012, 125(3):260-266.

10. Tran BX, Ohinmaa A, Duong AT, Do NT, Nguyen LT, Mills S, Houston $S$, Jacobs P: Cost-effectiveness of methadone maintenance treatment for HIV-positive drug users in Vietnam. AIDS Care 2012, 24(3):283-290.

11. Tran BX, Nguyen N, Ohinmaa A, Duong AT, Nguyen LT, Van Hoang M, Vu PX, Veugelers PJ: Prevalence and correlates of alcohol use disorders during antiretroviral treatment in injection-driven HIV epidemics in Vietnam. Drug Alcohol Depend 2013, 127(1-3):39-44.

12. Ezard N, Oppenheimer E, Burton A, Schilperoord M, Macdonald D, Adelekan M, Sakarati A, van Ommeren M: Six rapid assessments of alcohol and other substance use in populations displaced by conflict. Confl Health 2011, 5(1):1.

13. Neramitpitagkul P, Lertpitakpong C, Yothasamut J, Thavorncharoensap M, Chaikledkaew $U$, Teerawattananon Y: Economic impact on health-care costs related to major diseases including HIV/AIDS due to alcohol drinking among Thai populations. Value Health 2009, 12(Suppl 3):S97-S100.

14. le Tho H, Singhasivanon P, Kaewkungwal J, Kaljee LM, Charoenkul C: Sexual behaviors of alcohol drinkers and non-drinkers among adolescents and young adults in Nha Trang, Vietnam. Southeast Asian J Trop Med Public Health 2007, 38(1):152-160

15. Luo XF, Duan S, Duan QX, Pu YC, Yang YC, Wong FY, He N: Prevalence and correlates of alcohol use and subsequent sexual activity among adult males in a rural community of ethnic minorities in Yunnan Province, China. Biosci Trends 2012, 6(6):288-295.

16. Li Q, Li X, Stanton B: Alcohol use and sexual risk behaviors and outcomes in China: a literature review. AIDS Behav 2010, 14(6):1227-1236

17. Tran BX, Ohinmaa A, Duong AT, Nguyen LT, Vu PX, Mills S, Houston S, Jacobs $P$ : The cost-effectiveness and budget impact of Vietnam's methadone maintenance treatment programme in HIV prevention and treatment among injection drug users. Glob Public Health 2012, 7(10):1080-1094.

18. Tran DA, Shakeshaft A, Ngo AD, Mallitt KA, Wilson D, Doran C, Zhang L: Determinants of antiretroviral therapy initiation and treatment outcomes for people living with HIV in Vietnam. HIV Clin Trials 2013, 14(1):21-33.

19. Tran BX, Nguyen LT, Nguyen NH, Hoang QV, Hwang J: Determinants of antiretroviral treatment adherence among HIV/AIDS patients: a multisite study. Glob Health Action 2013, 6:19570.

20. DA C d, Thorson A, Sonnerborg Hoa NP, Chuc NT, Phuc HD, Larsson M: Survival and causes of death among HIV-infected patients starting 
antiretroviral therapy in north-eastern Vietnam. Scand J Infect Dis 2012, 44(3):201-208.

21. Tran BX, Ohinmaa A, Duong AT, Do NT, Nguyen LT, Nguyen QC, Mills S, Jacobs $P$, Houston S: Changes in drug use are associated with healthrelated quality of life improvements among methadone maintenance patients with HIV/AIDS. Qual Life Res 2012, 21(4):613-623.

22. Van Tam V, Larsson M, Pharris A, Diedrichs B, Nguyen HP, Nguyen CT, Ho $P D$, Marrone $G$, Thorson A: Peer support and improved quality of life among persons living with HIV on antiretroviral treatment: a randomised controlled trial from north-eastern Vietnam. Health Qual Life Outcomes 2012, 10:53.

23. Tran BX, Ohinmaa A, Nguyen LT, Nguyen TA, Nguyen TH: Determinants of health-related quality of life in adults living with HIV in Vietnam. AIDS Care 2011, 23(10):1236-1245.

24. Tran BX, Ohinmaa A, Nguyen LT: Quality of life profile and psychometric properties of the EQ-5D-5 L in HIV/AIDS patients. Health Qual Life Outcomes 2012, 10(1):132.

25. Tran BX, Nguyen NPT: Patient Satisfaction with HIV/AIDS Care and Treatment in the Decentralization of Services Delivery in Vietnam. PLoS One 2012, 7(10):e46680.

26. Giang KB, Allebeck P, Spak F, Van Minh H, Dzung TV: Alcohol use and alcohol consumption-related problems in rural Vietnam: an epidemiological survey using AUDIT. Subst Use Misuse 2008, 43(3-4):481-495.

27. Broyles LM, Gordon AJ, Sereika SM, Ryan CM, Erlen JA: Predictive Utility of Brief Alcohol Use Disorders Identification Test (AUDIT) for human immunodeficiency virus antiretroviral medication nonadherence. Subst Abus 2011, 32(4):252-261.

28. Giordano TP, Guzman D, Clark R, Charlebois ED, Bangsberg DR: Measuring adherence to antiretroviral therapy in a diverse population using a visual analogue scale. HIV Clin Trials 2004, 5(2):74-79.

29. WHOQOL HIV Group: WHOQOL-HIV for quality of life assessment among people living with HIV and AIDS: results from the field test. AIDS Care 2004, 16(7):882-889.

30. O'Connell K, Skevington S, Saxena S: Preliminary development of the World Health Organsiation's Quality of Life HIV instrument (WHOQOLHIV): analysis of the pilot version. Soc Sci Med 2003, 57(7):1259-1275.

31. Tran BX, Ohinmaa A, Nguyen LT, Oosterhoff P, Vu PX, Vu TV, Larsson M: Gender differences in quality of life outcomes of HIV/AIDS treatment in the latent feminization of HIV epidemics in Vietnam. AIDS Care 2012, 24(10):1187-1196.

32. Tran BX: Quality of life outcomes of antiretroviral treatment for HIV/AIDS patients in Vietnam. PLoS One 2012, 7(7):e41062.

33. Linden A, Adams JL: Evaluating health management programmes over time: application of propensity score-based weighting to longitudinal data. J Eval Clin Pract 2010, 16(1):180-185.

34. Lu B: Propensity score matching with time-dependent covariates. Biometrics 2005, 61(3):721-728.

35. Austin PC, Escobar M, Kopec JA: The use of the Tobit model for analyzing measures of health status. Qual Life Res 2000, 9(8):901-910.

36. Azar MM, Springer SA, Meyer JP, Altice FL: A systematic review of the impact of alcohol use disorders on HIV treatment outcomes, adherence to antiretroviral therapy and health care utilization. Drug Alcohol Depend 2010, 112(3):178-193.

37. Senbanjo R, Wolff $K$, Marshall J: Excessive alcohol consumption is associated with reduced quality of life among methadone patients. Addiction 2007, 102(2):257-263.

38. Tran BX, Ohinmaa A, Duong AT, Do NT, Nguyen LT, Nguyen QC, Mills S, Jacobs $P$, Houston $S$ : Changes in drug use are associated with healthrelated quality of life improvements among methadone maintenance patients with HIV/AIDS. Qual Life Res 2011, 21(4):613-623.

39. Tuunanen $M$, Aalto $M$, Seppa K: Binge drinking and its detection among middle-aged men using AUDIT, AUDIT-C and AUDIT-3. Drug Alcohol Rev 2007, 26(3):295-299.

40. Bradley KA, DeBenedetti AF, Volk RJ, Williams EC, Frank D, Kivlahan DR: AUDIT-C as a brief screen for alcohol misuse in primary care. Alcohol Clin Exp Res 2007, 31(7):1208-1217.
41. Frank D, DeBenedetti AF, Volk RJ, Williams EC, Kivlahan DR, Bradley KA: Effectiveness of the AUDIT-C as a screening test for alcohol misuse in three race/ethnic groups. J Gen Intern Med 2008, 23(6):781-787.

42. Mai Do Hoa MD, Pham Viet Cuong: ART adherence among people living with HIV/AIDS in Ha Noi and Hai Duong: situation and associated factors. J Pract Med, Hanoi 2010, 243\&243(2011):5.

doi:10.1186/1471-2458-14-27

Cite this article as: Tran et al:: Associations between alcohol use disorders and adherence to antiretroviral treatment and quality of life amongst people living with HIV/AIDS. BMC Public Health 2014 14:27.

\section{Submit your next manuscript to BioMed Central and take full advantage of:}

- Convenient online submission

- Thorough peer review

- No space constraints or color figure charges

- Immediate publication on acceptance

- Inclusion in PubMed, CAS, Scopus and Google Scholar

- Research which is freely available for redistribution 